\title{
Comportamiento de ovinos de ceba establecidos dentro de sistema herbáceo y árboles de pera (Pyrus communis)
}

\author{
Fattening sheep behavior established within herbaceous system and pear \\ trees (Pyrus communis) \\ Sánchez Vladimir ${ }^{1}$ y Ojeda F. ${ }^{2}$ \\ ${ }^{1} \mathrm{MVZ}, \mathrm{MSc}$ Docente Universidad de los Llanos \\ ${ }^{2} \mathrm{PhD}$, Estación Experimental de Pastos y Forrajes "Indio Hatuey" Central España \\ Republicana, CP 44280, Matanzas, Cuba \\ vladimirsanchez@unillanos.eu.co
}

Recibido 09 de Febrero 2015, Aceptado 10 de Abril 2015

\section{RESUMEN}

Debido a la creencia popular que los ovinos pueden ser depredadores de los árboles frutales, los productores de pera (Pyrus communis) no utilizan el estrato herbáceo de sus cultivos como áreas de pastoreo. El presente trabajo demostró que es posible pastorear en un sistema integrado (sistema agrosilvopastoril) dichas áreas sin riesgo alguno para los árboles, a la vez que se obtenían buenas ganancias de peso y se controlaba la vegetación espontánea.

Palabras clave: Silvopastoreo, frutales, ovinos, peras.

\begin{abstract}
Due to the popular belief that sheep can be predators of fruit trees, producers of pear (Pyrus communis) in Colombia, is not use their herbaceous stratum as grazing. This work demonstrated that is possible to graze those areas in an integrated system (agrosilvopastoral system) without risk to the pear trees, while that obtained good weight gain and weeds spontaneous was controlled.
\end{abstract}

Keywords: Silvopasture, fruit, sheep, pears. 


\section{RESUMO}

Devido à crença popular de que as ovelhas podem ser predadores de árvores de fruto, os produtores de pêra (Pyrus communis) não usam o estrato herbáceo de suas lavouras e áreas de pastagem. Este trabalho demonstrou que é possível para pastar em um sistema integrado (sistema agroflorestal) tais áreas sem qualquer risco para as árvores, enquanto bons ganhos de peso foram obtidos e vegetação espontânea foi controlada.

Palavras-chave: Silvopastoralismo, frutas, ovelhas, peras.

\section{INTRODUCCIÓN}

Entre las plantaciones de frutales caducifolios de trópico alto de Colombia no es usual el manejo de ovinos por la creencia popular por parte de los productores de que "la saliva de las ovejas acaba con todo lo que toca" sin que dicha afirmación tenga una base real pues interpreta al ovino como un depredador natural de todo lo verde, sin considerar que la corteza de los árboles no es un alimento natural en sus hábitos alimentarios, como puede ser el caso de los caprinos.

En igual sentido, es poca la información que disponible a nivel mundial sobre el silvopastoreo de ovinos en frutales, es importante señalar los trabajos de pastoreo dentro de plantaciones de cítricos en Cuba hechos por Simón et al., (2000; 2004); así como los de ovinos dentro de cítricos, realizados también en Cuba por Borroto et al., (1994), Mazorra et al., (2001; 2003; 2004); pastoreo en plantaciones de mango, informados por Ezcurra y Callejas, (1989) en México y por Combellas, (1997) en Venezuela; y pastoreo con ovinos dentro de cultivos de café, hechos en México por Durantes et al., (2004), Torres, (2004) y Dávila et al., (2004). Sánchez, (1995) planteó la posibilidad de explotar animales dentro de plantaciones de pera, durazno y manzana.

Con estos antecedentes se entendió que era posible la introducción de ovinos dentro de plantaciones de frutales caducifolios de clima frío, para lo cual se hizo necesario realizar una prueba etológica a nivel de campo para determinar la 
reacción de los ovinos frente a los árboles de pera y precisar si tenía fundamento o no, la creencia popular acerca del posible daño que hacen los ovinos a los árboles. De acuerdo a los estudios los objetivos de esta investigación fueron: determinar si los ovinos realmente atacan el follaje y la corteza de los árboles de pera y cuantificar la vegetación espontánea del estrato herbáceo y su aprovechamiento por los ovinos.

\section{METODOLOGÍA}

La investigación se realizó en la finca "La Armenia", del municipio de Nuevo Colón del Departamento de Boyacá en Colombia, ubicada a $5^{\circ} 21^{\prime}$ de latitud norte, a una altitud de $2.450 \mathrm{msnm}$, con temperatura promedio de $14^{\circ} \mathrm{C}$, precipitación anual de $900 \mathrm{~mm}$ (régimen de lluvias monomodal desde Abril hasta mediados de Diciembre) y humedad relativa del 70\% (Bernal y Cortés, 1993). De acuerdo con Salamanca, (1998) la clasificación ecológica de la región es de bosque seco montano bajo (bs-MB). El suelo se clasifica como Franco, con pH 5.2 y materia orgánica de $1.63 \%$.

El cultivo de pera utilizado tiene 20 años de establecido con un marco de siembra de $6 \times 5 \mathrm{~m}$ y un área total de 1.2 ha, aunque para esta investigación sólo se emplearon $3.630 \mathrm{~m}^{2}$, el período de evaluaciones tuvo una duración de 43 días, se utilizaron nueve ovinos de la raza criolla (seis hembras y tres machos) para una carga equivalente a 24.8 animales/ha, los cuales no tenían experiencia previa de pastoreo dentro de cultivos de pera, por lo que fueron manejados con cerca eléctrica de tres líneas en franjas $(6 \mathrm{~m})$ al ancho que formaban las hileras de pera (Figura 1). Para facilitar su traslado se utilizó una de las ovejas como guía, la cual permanecía todo el tiempo con un lazo amarrado al cuello.

Los animales tuvieron acceso a los árboles y al pasto que crece alrededor de estos (Figura 1), la zona de pastoreo dentro del peral fue cambiando en función de la disponibilidad de forraje, los ovinos tenían un peso promedio al inicio de $21 \mathrm{~kg}$ y fueron sacados cuando alcanzaron los $30 \mathrm{~kg}$. Para medir las ganancias, los animales fueron pesados cada 15 días. La prueba etológica tuvo una duración de 
20 días, con mediciones cada tercer día. Los animales se introducían al cultivo de pera desde las 8:30 a.m. hasta las 5:30 p.m.

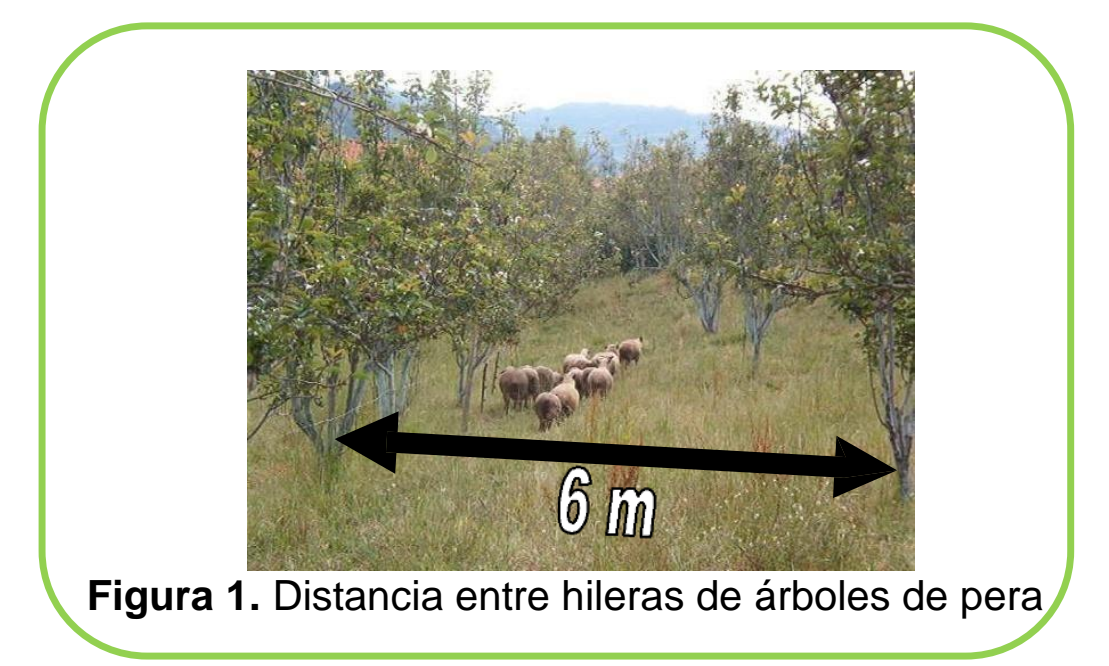

Las mediciones etológicas se hicieron cada hora a partir de las 9:00 a.m. y hasta las 5:00 p.m.; las variables tenidas en cuenta fueron: $C=$ Animal comiendo, $D=$ Animal desplazándose y $\mathrm{R}=$ Animal en reposo - rumiando. Diariamente se inspeccionó el área de pastoreo para verificar si había daños en el peral, se midió la disponibilidad de forraje mensualmente, siguiendo la metodología propuesta por Martínez et al., (1990) y la composición botánica a través del "método de los pasos", descrito por Anon, (1980).

\section{RESULTADOS Y DISCUSIÓN}

Composición botánica: En este ensayo los animales consumieron el estrato herbáceo espontáneo que crece dentro del peral después de la cosecha. Las especies encontradas se muestran en el cuadro 1. El pasto predominante durante todo el período de ensayo (Gráfico 1) fue el Ray grass (Lolium sp.), siguiéndole en proporción la gualola ( $P$. segetum) y el rábano amarillo (Brassica $s p$.).

El rábano morado ( $R$. raphanistrum), el carretón cadillo ( $M$. hispida) y el bledo (Amaranthus $s p$ ) iniciaron el ensayo con proporciones similares, pero el rábano morado aumentó en el tiempo; mientras que el carretón cadillo se mantuvo y el bledo tendió a desaparecer. En el caso de la avena ( $A$. fatua) no sufrió cambios durante el período evaluado. La prueba permitió corroborar que el Ray grass, 
además de ser predominante, tiene la capacidad de resistir el pisoteo, servir de alimento a los animales y ser el forraje que mejor se adapta a estas condiciones edafoclimáticas.

Cuadro 1. Variedad del estrato herbáceo en la fase de Etología

\begin{tabular}{cc}
\hline Nombre común & Nombre científico \\
\hline Ray grass & Lolium $s p$. \\
Gualola & Polygonum segetum \\
Rábano amarillo & Brassica sp. \\
Rábano morado & Raphanus raphanistrum \\
Carretón cadillo & Medicago hispida \\
Bledo & Amaranthus $s p$. \\
Avena silvestre & Avena fatua \\
Cerraja & Sonchus olenaceus $(L)$ \\
\hline
\end{tabular}

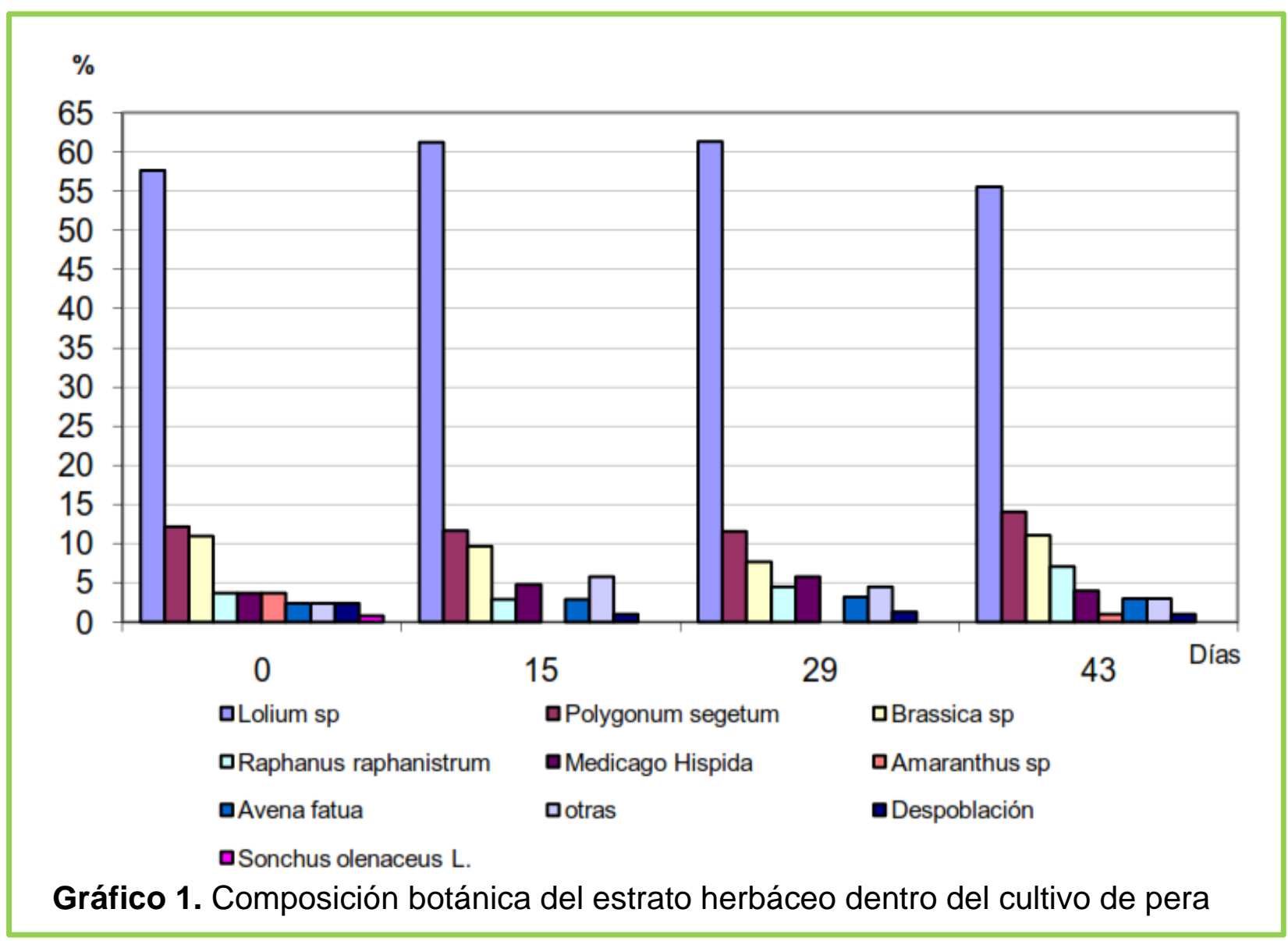

Lo anterior está en concordancia con lo hallado en condiciones de climas templados por Mosquera et al., (2000) quienes consideran que además de 
presentar un buen valor nutritivo, resulta un pasto muy agresivo, capaz de controlar la invasión de otras especies espontáneas que puedan competir con él.

El carretón cadillo ( $M$. hispida), también conocido como trébol de California (Berlijn y Bernardon, 2001), resulto ser muy palatable para las ovejas. Por el hecho de ser una leguminosa fijadora de nitrógeno, contribuye a mejorar la calidad del suelo y proporciona, además, un aporte apreciable de proteína para los animales. El mayor inconveniente que se le señala es que sus frutos se adhieren a la lana de las ovejas, pero su presencia en una asociación con gramínea lo convierte en una opción aceptable como estrato herbáceo.

La avena ( $A$. fatua) es una planta bien consumida por los animales y, como el pH del terreno es óptimo para su desarrollo, permanece de forma espontánea dentro de los pastoreos. Esta especie contribuye a aportar una parte de la proteína extra que reciben los animales dentro del pastizal. No obstante sus bondades como forraje, no es recomendable como principal cultivo, a menos que se cuente con riego, por ser uno de los cereales que más agua consumen por kilogramo de materia seca producido (Salamanca, 1998), acción que puede perjudicar la absorción de humedad por parte de los árboles.

Disponibilidad: Danelón et al., (2001) consideran que es preferible utilizar un método para medir la disponibilidad de forraje que requiere tomar un gran número de observaciones pero en forma rápida y sencilla, al costo de una aceptable menor precisión, a otros más precisos y sofisticados, como puede ser el caso de los que basan su funcionamiento en la capacitancia electrónica. A conclusiones similares llegaron Paciullo et al., (2004) al estudiar el método del disco. Por esta razón el método utilizado fue el de Martínez et al., (1990). Durante todo el período evaluativo se garantizó que la disponibilidad siempre fuera alta (cuadro 2), de forma tal que el pasto no fuera limitante, lo cual permitió que los animales pudieran manifestar sus hábitos alimenticios sin perturbaciones, al comienzo del ensayo dicha disponibilidad fue de 4.32 ton de MS/ha. 
Cuadro 2. Disponibilidad de forraje

\begin{tabular}{cc}
\hline Medición & Disponibilidad (Ton MS/ha) \\
\hline 1 & 4.32 \\
2 & 3.91 \\
\hline
\end{tabular}

Esta premisa resulta de gran importancia, ya que Castro et al., (1993) hallaron pérdidas de peso en las ovejas cuando pastoreaban sin suplementación una pradera de Trifolium repens, Lolium perenne y Festuca pratensis, con poca disponibilidad. Igualmente, las investigaciones desarrolladas por Osoro y Martínez, (1993) con ovinos que pastaban praderas con pastos de porte muy bajo, indican que los animales presentaron pérdidas de peso y una baja en su condición corporal. Al final del experimento la disponibilidad bajó a 3.91 ton de $\mathrm{MS} / \mathrm{ha}$, lo que corrobora la idea que los ovinos son una herramienta útil en el control de los arvenses dentro de plantaciones de pera.

Etología: El comportamiento etológico de los animales durante el ensayo se muestra en el Gráfico 2.

En la evaluación se constató que los animales realizaron el mayor consumo de forraje en las primeras horas de pastoreo y al final de la tarde. También se observó un mayor número de animales en reposo-rumiando en las horas con mayor intensidad lumínica y temperatura, entre la 1:00 p.m. y las 3:00 p.m. Estos resultados coinciden con los hallados por Mazorra et al., (2003) en plantaciones de cítricos y con los de Baumont et al., (2000) en condiciones de pastoreo para esta especie. Durante el período evaluativo no se detectaron acciones depredativas de los animales hacia las hojas o la corteza de los árboles

Rendimiento animal: Con respecto al comportamiento zootécnico, los ovinos presentaron una buena respuesta, con ganancias de peso de 209 g/animal/día (Gráfico 3), las cuales pueden considerarse como excelentes si se comparan con las halladas por Osoro y Martínez, (1993) que sólo fueron de 87 g/animal/día. 


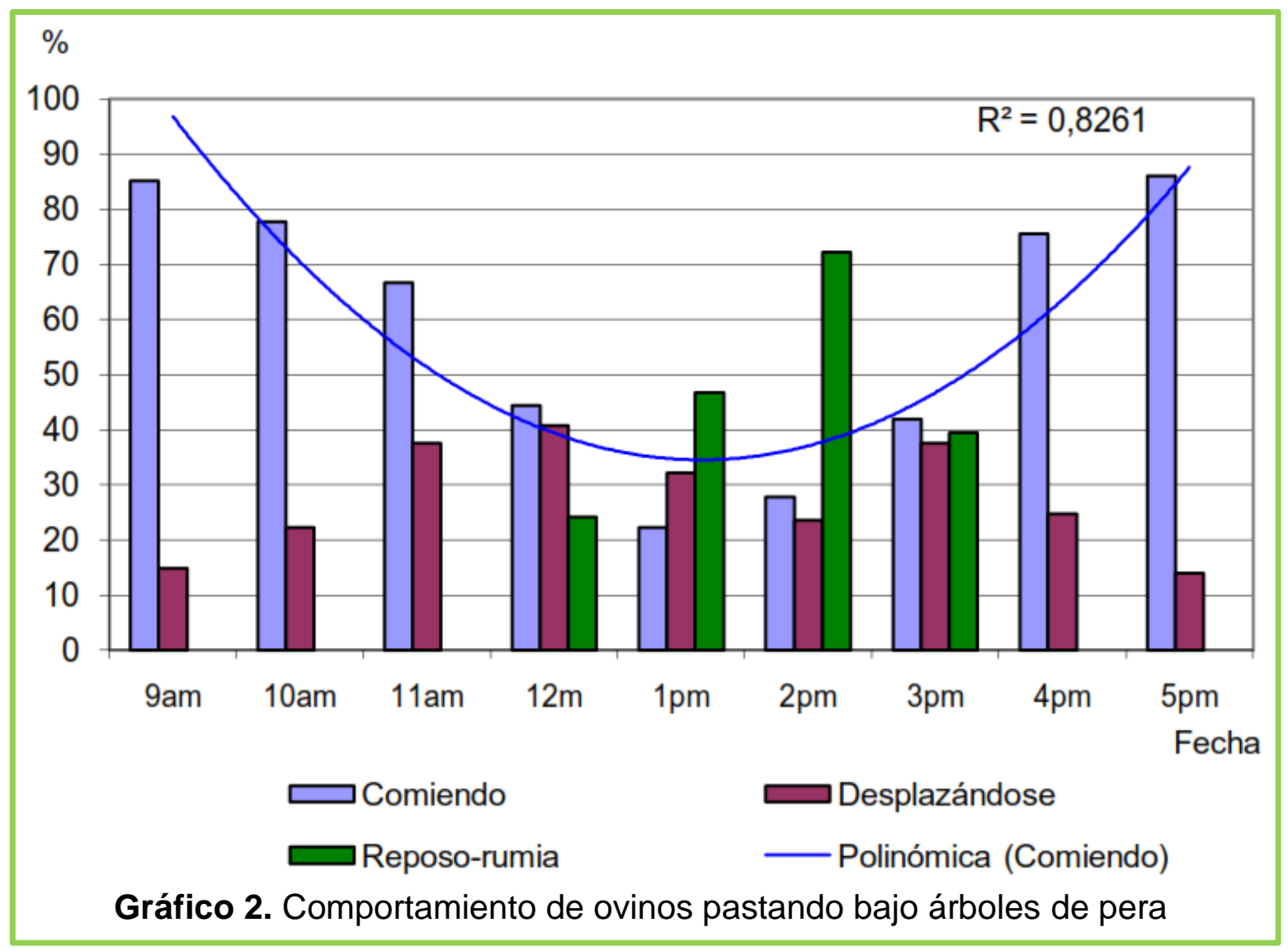

Los valores hallados también están por encima de los informados por otros autores en condiciones de trópico bajo, como Arcos et al., (2002), quienes encontraron ganancias de $17.3 \mathrm{~g} / \mathrm{animal} / \mathrm{día}$ para las hembras de 6 meses de edad, $22.1 \mathrm{~g} /$ animal/día para los machos de siete meses de edad y 85.0 y 88.5 g/animal/día para hembras y machos de levante, respectivamente. Clavero, (1998) en el trópico bajo, reporta ganancias entre 7.5 y $27.6 \mathrm{~g} / \mathrm{animal} / \mathrm{día}$ en corderos suplementados con diferentes niveles de maíz (Zea maiz) y cují (Prosopis juliflora).

En igual sentido, estos resultados indican que existe un gran potencial productivo para los ovinos bajo las condiciones de trópico alto, dado fundamentalmente por la calidad del forraje consumido.

En la respuesta encontrada incidieron además, como factores determinantes, la alta disponibilidad de pasto en el área y la posible ocurrencia de un fuerte crecimiento compensatorio. 


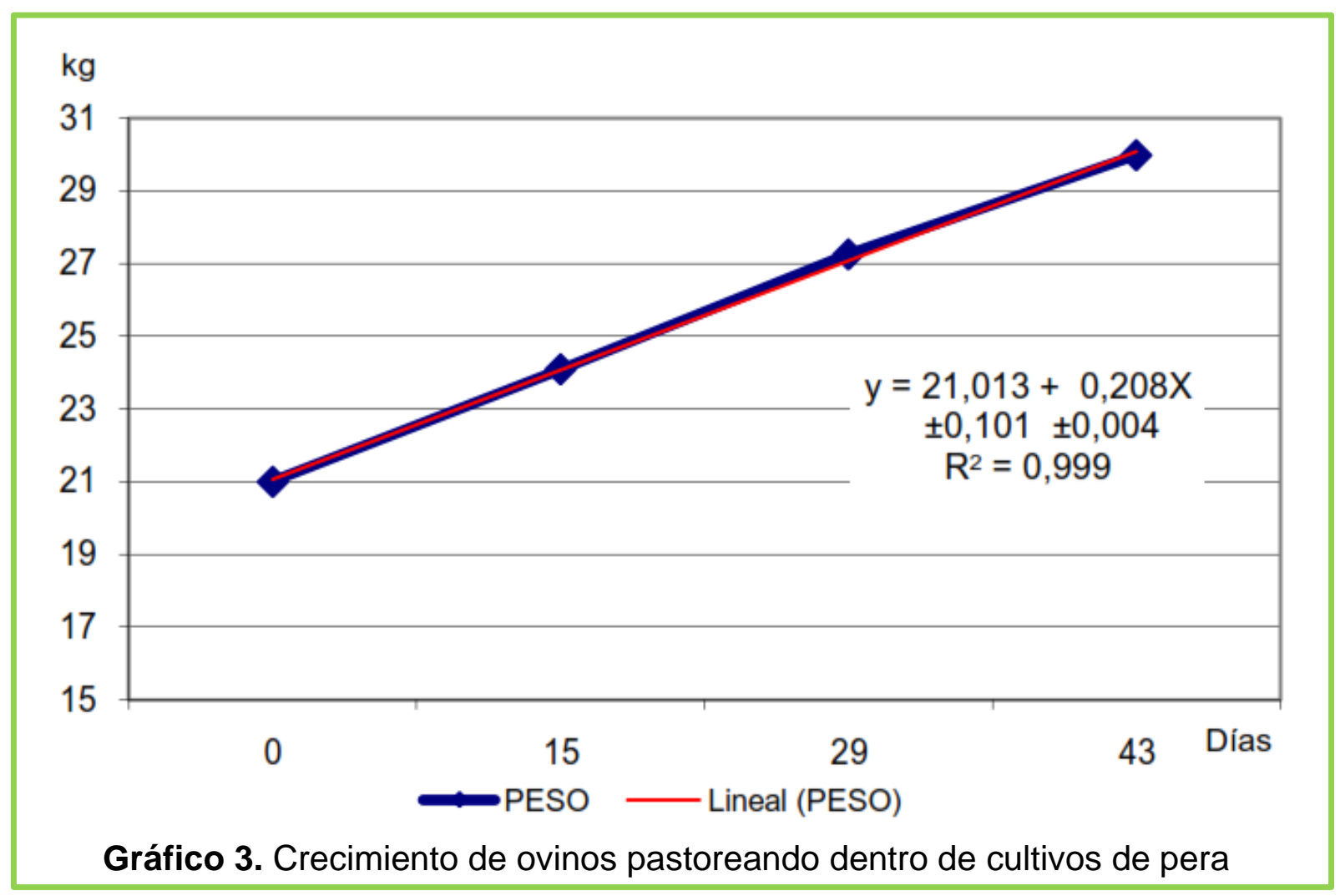

\section{CONCLUSIONES}

De estos resultados se concluyó que era factible introducir ovinos en un cultivo de pera, sin riesgo a que comprometiesen la estabilidad de la plantación; que los animales son capaces de controlar el estrato herbáceo dentro de la plantación; que se obtenían ganancias de peso adecuadas y que sería conveniente realizar una siembra dirigida de pasto de buena calidad para garantizar que la disponibilidad de forraje no quedara en manos de la hierba que crece espontáneamente; además, se demostró que el Ray grass es la gramínea que crece con mayor agresividad dentro de este cultivo. En igual sentido, los resultados señalaron la conveniencia de introducir una leguminosa, que además de aportar nitrógeno al suelo, permitiera mejorar la base alimentaria de los ovinos.

\section{REFERENCIAS BIBLIOGRÁFICAS}

1 Anon. Muestreo de Pastos. Estación Experimental de Pastos y Forrajes "Indio Hatuey", Taller del IV Seminario científico de la EEPF "Indio Hatuey", Matanzas, Cuba, 35 p. 1980. 
2 Arcos J, Romero H, Vanegas M, Riveros E. Ovinos Colombianos de Pelo. Alternativa productiva para el sur del departamento del Tolima, Ed Tecnimpresos, Colombia. CORPOICA, PRONATTA, 80 p. 2002.

3 Baumont R, Prache S, Meuret M, Morand-Fehr P. How forages characteristics influence behaviour and intake in small ruminants: a Review. Livestock Production Science, 64 (1): 15-28. 2000.

4 Berlijn J, Bernardon A. Pastizales Naturales. México. Secretaría de Educación Pública SEP. Ed. Trillas, 80 p. 2001.

5 Bernal ER, Cortés OI. Atlas temático del municipio de Nuevo Colón. Tunja. Licenciado en Ciencias Sociales y Económicas, Universidad Pedagógica y Tecnológica de Colombia. Facultad de Ciencias de la Educación, Escuela de Ciencias Sociales y Economía. 125 p. 1993.

6 Borroto A, Pérez R, Hernández N, Bacallao J. Sustitución del suplemento proteico en ceba ovina con el uso de banco de proteína. Rev. Cub. Prod. Anim., 8 (1): 20-23. 1994.

7 Castro T, Manso T, Mantecón A. Respuesta a la suplementación en pastoreo de ovejas de raza Churra en lactación: Ingestión y rendimientos productivos. XXV Jornadas de Estudio AIDA (V Jornadas sobre Producción Animal). ITEA, 12 (1): 63-65. 1993.

8 Clavero T. El Cují (Prosopis juliflora), Serie Técnica: Árboles Forrajeros 3. Maracaibo. Centro de Transferencia de Tecnología en Pastos y Forrajes, La Universidad del Zulia, Maracaibo, Venezuela. 1998.

9 Combellas J. Producción de ovinos en Venezuela. Fundación Polar, Universidad Central de Venezuela, Ed Arte. 62 p. 1997.

10 Danelón J, Dapuente C, Jaurena G, Cantet R, Saucede M. Eficiencia de la capacitancia y altura de canopeo comprimido (con disco) para estimar biomasa forrajera. Rev. Facultad de Agronomía, 21 (3): 213-219. 2001.

11 Dávila SP, Sanginés GL, Acevedo JA, Pérez RF. Comportamiento productivo y etológico de ovinos en pastoreo de arvenses de cafetal comparado con gramíneas con y sin suplementación y su efecto en la digestibilidad in situ. Departamento de Producción Vegetal. Escuela Politécnica Superior. Universidad Santiago de Compostela, Silvopastoralismo y Manejo Sostenible. Congreso Internacional. Lugo, España. 163 p. 2004.

12 Durantes CA, Torres JA, Martínez PA, Castro R, Arroyo A, Cruz J. Empleo de cloruro de litio y jarabe de ipecacuana como inductores de la aversión de las ovejas a consumir las plantas de café. En: Departamento de Producción Vegetal. Escuela Politécnica Superior. Universidad Santiago de Compostela, Silvopastoralismo y Manejo Sostenible. Congreso Internacional, Lugo, España. 69 p. 2004.

13 Ezcurra L, Callejas A. Producción de ganado ovino en la América Tropical y el Caribe. Ed CIDA, La Habana, Cuba. 233 p. 1989.

14 Martínez J, Milera M, Remy V, Yepes I, Hernández J. Un método ágil para estimar la disponibilidad de pasto en una vaquería comercial. Pastos y Forrajes, 13 (1): 101-110. 1990.

15 Mazorra C, de la Rosa A, Álvarez A, Borroto A. Consideraciones sobre los sistemas de producción de ovinos y sus potencialidades para Cuba. Pastos y Forrajes, 24 (1): 81-90. 2001. 
16 Mazorra C, Borroto A, Pérez R, Fontes D, Borges G. Potencialidades de los subproductos citrícolas en la alimentación de los ovinos. Principales Sistemas de Alimentación. 2003. Recuperado 15 noviembre 2003. Disponible En: http://www.cirval.asso.fr/publication/venezuela/Conferencias/Potencialidades.h $\underline{\operatorname{tm}}$

17 Mazorra C, Borroto A, de La Rosa A, Pérez R, López J. El Consumo de forrajes: Alternativa para disminuir el ramoneo de ovinos pastoreando en plantaciones citrícolas. 2003. Recuperado 16 noviembre 2003. Disponible En: http://www.cipav.org.co/redagrofor/memorias99/Mazorra.htm

18 Mazorra C, Provenza FD, Borges G, Blanco M. Principios prácticos para integrar ovinos en pastoreo en los ecosistemas citrícolas en Cuba. 2004. En: Departamento de Producción Vegetal. Escuela Politécnica Superior. Universidad Santiago de Compostela, Silvopastoralismo y Manejo Sostenible. Congreso Internacional, Lugo, España. 90 p. 2004.

19 Mosquera M, Romero R, Rigueiro A, González M, López L. Plantas de los prados del noreste de la Península Ibérica. Universidad de Santiago de Compostela, Escola Politécnica Superior, Santiago de Compostela, España. 111 p. 2000.

20 Osoro K, Martínez A. Efecto de la altura del pasto disponible en praderas mejoradas con Raygras y Trébol sobre la producción ovina. En: XXV Jornadas de Estudio AIDA (V Jornadas sobre Producción Animal). ITEA, 12 (1): 57-59. 1993.

21 Paciullo DS, Aroeira LJM, Cóser AC, Cardoso RC. Uso do Método do disco e da altura da planta para estimar a massa de forragem em relvado de Cynodon ssp. Ciência Rural, 34 (2): 599-601. 2004.

22 Salamanca R. Pastos y Forrajes - Producción y Manejo. Universidad Santo Tomás, Colombia. 343 p. 1998.

23 Sánchez MD. Integration of Livestock with Perennial Crops. En: World Animal Review. FAO. 82 (1): 50-57. 1995.

24 Simón L, Sánchez MD, Hernández M, Sánchez S y Mendoza C. Integración de equinos en plantaciones de naranjos (Citrus sinensis). 2000. [Disco]. Consultado 11 noviembre 2000. En: I Conferencia Electrónica Nacional sobre Sistemas Silvopastoriles.

25 Simón L, Sánchez MD, Hernández M, Sánchez S y Mendoza C. Integración de equinos en plantaciones de naranjos. En: Departamento de Producción Vegetal. Escuela Politécnica Superior. Universidad Santiago de Compostela, Silvopastoralismo y Manejo Sostenible. Congreso Internacional. Lugo, España. 107 p. 2004.

26 Torres JA. Efecto del pastoreo con ovinos en la calidad del café. En: Departamento de Producción Vegetal. Escuela Politécnica Superior. Universidad Santiago de Compostela, Silvopastoralismo y Manejo Sostenible. Congreso Internacional. Lugo, España. 107 p. 2004.

27 Torres JA. Sistema silvopastoral café-ovinos en Veracruz, México. En: Departamento de Producción Vegetal. Escuela Politécnica Superior. Universidad Santiago de Compostela, Silvopastoralismo y Manejo Sostenible. Congreso Internacional. Lugo, España. 107 p. 2004. 